\title{
Significant Association of Alpha-Methylacyl-CoA Racemase Gene Polymorphisms with Susceptibility to Prostate Cancer: a Meta-Analysis
}

\author{
Nan Chen $^{1 \&}$, Jia-Rong Wang ${ }^{1 \&}$ Lin Huang ${ }^{1 \&}$, Yang Yang1, Ya-Mei Jiang ${ }^{1}$, Xiao- \\ Jiang Guo', Ya-Zhou He ${ }^{1,2}$, Yan-Hong Zhou ${ }^{3 *}$
}

\begin{abstract}
Background: Alpha-methylacyl-CoA racemase(AMACR) is thought to play key roles in diagnosis and prognosis of prostate cancer. However, studies of associations between AMACR gene polymorphisms and prostate cancer risk reported inconsistent results. Therefore, we conducted the present meta-analysis to clarify the link between AMACR gene polymorphisms and prostate cancer risk. Materials and Methods: A literature search was performed in PubMed, Embase, China National Knowledge Infrastructure (CNKI), Wanfang and Weipu databases. Odds ratios (ORs) and $95 \%$ confidence intervals $(95 \% \mathrm{CIs})$ were calculated to assess the strength of any association between AMACR polymorphisms and prostate cancer risk. Subgroup analyses by ethnicity, source of controls, quality control and sample size were also conducted. Results: Five studies covering 3,313 cases and 3,676 controls on five polymorphisms (D175G, M9V, S201L, K277E and Q239H) were included in this meta-analysis. Significant associations were detected between prostate cancer and D175G (dominant model: $\mathrm{OR}=0.89,95 \% \mathrm{CI}=\mathbf{0 . 8 0 - 0 . 9 9}, \mathrm{P}=\mathbf{0 . 0 4}$ ) and $\mathrm{M} 9 \mathrm{~V}$ (dominant model: $\mathrm{OR}=0.87,95 \% \mathrm{CI}=\mathbf{0 . 7 8 - 0 . 9 7 ,} \mathrm{P}=0.01$ ) polymorphisms as well as that in subgroup analyses. We also observed significant decreased prostate cancer risk in the dominant model $(\mathrm{OR}=0.90,95 \% \mathrm{CI}=0.81-0.99, \mathrm{P}=0.04)$ for the $\mathrm{S201 \textrm {L }}$ polymorphism. However, $\mathrm{K} 277 \mathrm{E}$ and Q239H polymorphisms did not appear to be related to prostate cancer risk. Conclusions: The current metaanalysis indicated that $\mathrm{D175G}$ and $\mathrm{M9V}$ polymorphisms of the AMACR gene are related to prostate cancer. The S201L polymorphism might also be linked with prostate cancer risk to some extent. However, no association was observed between K277E or Q239H polymorphisms and susceptibility to prostate cancer.
\end{abstract}

Keywords: Prostate cancer - AMACR - polymorphism - meta-analysis

Asian Pac J Cancer Prev, 16 (5), 1857-1863

\section{Introduction}

Prostate cancer $(\mathrm{PCa})$ is one of the most common malignancies in men with approximately 0.9 million newly diagnosed cases and 0.26 million deaths in 2008 worldwide (Jemal et al., 2011). Until now, the exact carcinogenic mechanism of prostate cancer has remained unclear (Mahmoud et al., 2014). It is widely accepted that carcinogenesis of prostate cancer is affected by genetic susceptibility and environmental exposure, for instance, a fatty acid diet (Amankwah et al., 2012; Doolan et al., 2014; Askari et al., 2014). Single nucleotide polymorphisms (SNPs), as a type of genetic predisposing factor, might lead to changes in protein encoding and be involved in the alterative risk of prostate cancer (Yang et al., 2013; Zhang et al., 2014). Therefore, it is crucial to focus on these genetic markers for their application in susceptibility prediction, early diagnosis and individualized therapeutic regimens of prostate cancer.
Alpha-methylacyl-CoA racemase (AMACR) is an important enzyme involved in the metabolism of branchedchain fatty acids as well as some drugs (Ferdinandusse et al., 2000). In a key chiral inversion step, R-2-methyl fatty acids is converted to corresponding acyl-CoA esters and subsequently to S-2-methylacyl-CoA ester via catalysis of AMACR (Lloyd et al., 2013). It is worth emphasizing that the change of expression and activity of AMACR might lead to low or high rate of fatty acids metabolism and the accumulation or reduction of R-2-methyl fatty acids in quantity, which ultimately caused diseases (Lloyd et al., 2013; Jiang et al., 2013). It was also identified that the overexpression of AMACR may play roles in stimulating prostate cancer cell growth through a pathway independent of androgen signaling (Zha et al., 2003). Furthermore, the concentration of this enzyme was observed to be much higher in prostate cancers than normal tissues and now AMACR is well used as an alternative biomarker of prostate specific antigen (PSA) for prostate cancer (Wang

${ }^{1}$ West China School of Medicine, ${ }^{2}$ Department of Gastrointestinal Surgery, ${ }^{3}$ Department of Laboratory Medicine, West China Hospital, Sichuan University, Chengdu, China ${ }^{\circledR}$ Equal contributors *For correspondence: Yanhong_zhou2013@163.com 
et al., 2009; Mazzola et al., 2011).

The AMACR gene was located on chromosome $5 \mathrm{p} 13$ and contained many common polymorphisms (D175G rs10941112, M9V rs3195676, S201L rs2287939, K277E rs2278008 and Q239H rs34677) (Daugherty et al., 2007; FitzGerald et al., 2008; Ouyang et al., 2011). Different polymorphisms of this gene might cause various concentration and activities of AMACR and resulted in various risk of prostate cancer (Zha et al., 2003; Ouyang et al., 2011; Lloyd et al., 2013). Up to now, a number of studies have been launched to investigate the association between AMACR gene polymorphisms and prostate cancer risk (Zheng et al., 2002; Daugherty et al., 2007; FitzGerald et al., 2008; Wright et al., 2011; Lee et al., 2013).

However, inconclusive findings were obtained from these studies, which may due to small research sample sizes and differences in ethnicity of study population. Thus, we performed the first meta-analysis with all published casecontrol studies available now to comprehensively evaluate the exact relationship of AMACR gene polymorphisms and susceptibility of prostate cancer. Each SNP we selected (D175G rs10941112, M9V rs3195676, S201L rs2287939, K277E rs2278008 and Q239H rs34677) was the one in which at least three studies were involved, to enhance the strength of evaluation of the association.

\section{Materials and Methods}

\section{Search strategy}

PubMed, Embase, Chinese National Knowledge Infrastructure (CNKI), Wanfang and Weipu (VIP) databases were searched (update to 2014.10.9) with combination of the following terms: "AMACR" or "Alpha-methylacyl-CoA racemase", "polymorphism", "mutation" or "variant" and "prostate cancer", "prostate carcinoma", or "PCa". There was no language restriction. Moreover, the reference lists of relative studies were also searched manually for potential case-control studies.

\section{Inclusion and Exclusion Criteria}

The inclusion criteria were as follows: (1) they were case-control studies; (2) the association between AMACR gene polymorphisms and prostate cancer risk was investigated; (3) detailed information of genotype frequency distribution was available for evaluating odds radio (OR) with $95 \%$ confidence interval (95\%CI). Studies were excluded if meeting the following criteria: (1) they were conference abstracts or reviews; (2) they were animal or cell line studies; (3) when more than two studies published duplicate data, studies with less sample size were excluded.

\section{Data extraction}

From each study, the following data were extracted: the name of first author, publication year, country, ethnicity of patients, source of controls (hospital based or population based), matching criteria for controls, number of cases and controls, genotyping method, and genotype frequency distribution of cases and controls. With a standard data extraction table, two investigators (Nan Chen and Lin
Huang) independently extracted data from each study. When disagreements arose, these two investigators launched a final discussion to make a consistent decision.

\section{Statistical analysis}

To estimate the Hardy-Weinberg equilibrium (HWE) for controls of each polymorphism in each study, we performed Person's $\mathrm{X}^{2}$ test and $\mathrm{P}$ value less than 0.05 was regarded as statistically significant. Data with disequilibrium in HWE in controls were excluded from further analysis. For assessing the association between AMACR polymorphisms and prostate cancer risk, the odds radio (ORs) with $95 \%$ CI were calculated under comparison of five genetic models: dominant model, recessive model, allel model, homozygote comparison, heterozygote comparison. The Z-test was conducted to evaluate the significance of the ORs and $\mathrm{P}<0.05$ was considered to be significant difference. We also performed $\mathrm{X}^{2}$ based $\mathrm{Q}$ and $\mathrm{I}^{2}$ test to assess heterogeneity among the studies and regarded it as statistical significance if $\mathrm{P}<0.1$. When significant heterogeneity was observed, the randomeffects model was applied to pool the data; Otherwise, the fixed-effects model was used. To avoid bias from different factors and backgrounds, we carried out subgroup analysis stratified by ethnicity, source of control, quality control and sample size. Visual inspection of potential asymmetry in funnel plots was used to estimate possible publication bias, and it was further quantitatively evaluated by Egger's test. For sensitivity analysis, we sequentially excluded each study one by one on the software and assessed the stability of the results. All analyses were performed with software Review Manager 5.2.

\section{Results}

\section{Characteristics of the eligible studies}

As shown in Figure 1, the search identified a total of 47 relative articles. After two reviewers (Nan Chen and Jia-Rong Wang) scanned the titles and abstracts independently based on the inclusion and exclusion criteria, 40 articles were excluded. Moreover, 2 articles were excluded when full-text articles were assessed. Finally, 5 studies with a total of 3313 cases and 3676 controls were included in this meta-analysis. Among them, there were 5 studies on D175G (rs10941112) and M9V (rs3195676) polymorphisms, 4 studies on S201L (rs2287939) and K277E (rs2278008) polymorphisms and 3 studies on Q239H (rs34677) polymorphism. Still, 4 studies included Caucasians, 1 study included Asians and 1 study included American Africans. There were 4 studies recruiting population-based controls while 1 studies recruiting hospital-based controls. The detailed information of included studies was presented in Table 1.

\section{Evidence synthesis}

The summary of genotypic distribution frequency of all five polymorphisms was presented in Table 2 and the results of meta-analysis were shown in Table 3. No between-study heterogeneity was detected and fix-effects model was applied to all analyses.

D175G polymorphism (rs 10941112) 
There were 5 studies with 3313 cases and 3676 controls involved in this polymorphism. Overall, significant association was observed between decreased risk of prostate cancer and D175G polymorphism (Dominant Model: $\mathrm{OR}=0.89,95 \% \mathrm{CI}=0.80-0.99, \mathrm{P}=0.04$ ) (Figure 2). In the subgroup analysis by ethnicity, we detected significant decreased prostate cancer risk in $\mathrm{GG}$ genotype among Caucasians (Dominant Model: $\mathrm{OR}=0.88$, 95\% CI=0.79-0.99, $\mathrm{P}=0.03$ ) (Figure 3). When stratified by source of controls, quality control and sample size, significant difference was also observed in studies with population-based controls (Dominant Model: $\mathrm{OR}=0.88$, 95\% CI=0.79-0.99, $\mathrm{P}=0.03$ ), quality control (Dominant Model: $\mathrm{OR}=0.89,95 \% \mathrm{CI}=0.79-1.00, \mathrm{P}=0.04)$ and sample size more than 500 (Dominant Model: $\mathrm{OR}=0.89$, $95 \% \mathrm{CI}=0.79-1.00, \mathrm{P}=0.04)$.

M9V polymorphism (rs3195676)

\section{Table 1. Baseline Characteristics of Eligible Case-control Studies}

\begin{tabular}{lrlllcccc}
\hline First Author & \#* & Year & Country & Ethnicity & $\begin{array}{c}\text { Source of } \\
\text { controls }\end{array}$ & Cases/Controls & $\begin{array}{c}\text { Quality } \\
\text { control }\end{array}$ & Polymorphisms \\
\hline Daugherty & 1 & 2007 & USA & Caucasian & PB & $1150 / 1381$ & Yes & D175G, M9V, S201L, K277E, Q239H \\
& 2 & 2007 & USA & African American PB & $102 / 396$ & Yes & D175G, M9V, S201L, K277E, Q239H \\
FitzGerald & 2008 & Australia & Caucasian & PB & $414 / 319$ & Yes & D175G, M9V \\
Lee & 2013 & Korea & Asian & HB & $194 / 168$ & NA & D175G, M9V, S201L, K277E, Q239H \\
Wright & 2011 & USA & Caucasian & PB & $1253 / 1249$ & Yes & D175G, M9V, S201L, K277E \\
Zheng & 2002 & USA & Caucasian & PB & $229 / 189$ & NA & D175G, M9V, S201L, K277E, Q239H \\
\hline
\end{tabular}

** number of data separately reported by articles; PB: population-based; HB: hospital-based; NA: not available

Table 2. Genotype Frequency Distribution of AMACR Polymorphisms

\begin{tabular}{|c|c|c|c|c|c|c|c|c|c|c|c|c|}
\hline \multirow{2}{*}{$\begin{array}{l}\text { Polymorphisms } \\
\text { D175G }\end{array}$} & \multirow{2}{*}{ First Author } & \multirow[t]{2}{*}{ \#* } & \multicolumn{4}{|c|}{ Case } & \multicolumn{4}{|c|}{ Control } & \multirow[t]{2}{*}{ HWE } & \multirow{2}{*}{$\begin{array}{l}\text { MAF in } \\
\text { controls }\end{array}$} \\
\hline & & & $\mathrm{N}$ & $\overline{\mathrm{AA}}$ & $\overline{A G}$ & GG & $\mathrm{N}$ & AA & $\mathrm{AG}$ & GG & & \\
\hline \multirow[t]{6}{*}{$(\mathrm{rs} 10941112)$} & Daugherty & 1 & 1135 & 330 & 562 & 243 & 1370 & 379 & 671 & 320 & YES & 0.48 \\
\hline & & 2 & 102 & 3 & 25 & 74 & 393 & 11 & 84 & 298 & YES & 0.87 \\
\hline & FitzGerald & & 414 & 125 & 197 & 92 & 319 & 74 & 155 & 90 & YES & 0.53 \\
\hline & Lee & & 194 & 29 & 96 & 69 & 168 & 26 & 66 & 76 & YES & 0.65 \\
\hline & Wright & & 1240 & 370 & 870 & & 1238 & 343 & 895 & & NA & NA \\
\hline & Zheng & & 228 & 76 & 100 & 52 & 188 & 56 & 82 & 50 & YES & 0.48 \\
\hline M9V & & & $\mathrm{N}$ & AA & $\mathrm{AG}$ & GG & $\mathrm{N}$ & AA & AG & GG & & \\
\hline \multirow[t]{6}{*}{ (rs3195676) } & Daugherty & 1 & 1124 & 329 & 560 & 235 & 1352 & 368 & 670 & 314 & YES & 0.48 \\
\hline & & 2 & 101 & 7 & 39 & 55 & 385 & 22 & 129 & 234 & YES & 0.78 \\
\hline & FitzGerald & & 414 & 128 & 197 & 89 & 318 & 76 & 151 & 91 & YES & 0.52 \\
\hline & Lee & & 192 & 29 & 94 & 69 & 168 & 26 & 68 & 74 & YES & 0.64 \\
\hline & Wright & & 1235 & 371 & 864 & & 1237 & 340 & 897 & & NA & NA \\
\hline & Zheng & & 226 & 74 & 103 & 49 & 182 & 53 & 79 & 50 & YES & 0.49 \\
\hline S201L & & & $\mathrm{N}$ & $\mathrm{CC}$ & $\mathrm{CT}$ & TT & $\mathrm{N}$ & $\mathrm{CC}$ & $\mathrm{CT}$ & TT & & \\
\hline \multirow[t]{5}{*}{ (rs2287939) } & Daugherty & 1 & 1110 & 575 & 441 & 94 & 1377 & 682 & 554 & 141 & YES & 0.3 \\
\hline & & 2 & 100 & 65 & 29 & 6 & 393 & 249 & 131 & 13 & YES & 0.2 \\
\hline & Lee & & 194 & 149 & 39 & 6 & 168 & 124 & 41 & 3 & YES & 0.14 \\
\hline & Wright & & 1253 & 636 & 617 & & 1249 & 598 & 651 & & NA & NA \\
\hline & Zheng & & 227 & 128 & 81 & 18 & 177 & 93 & 67 & 17 & YES & 0.29 \\
\hline K277E & & & $\mathrm{N}$ & AA & $\mathrm{AG}$ & GG & $\mathrm{N}$ & AA & $\mathrm{AG}$ & GG & & \\
\hline \multirow[t]{5}{*}{ (rs2278008) } & Daugherty & 1 & 1150 & 655 & 417 & 78 & 1381 & 756 & 507 & 118 & NO & 0.27 \\
\hline & & 2 & 102 & 65 & 32 & 5 & 396 & 254 & 127 & 15 & YES & 0.2 \\
\hline & Lee & & 194 & 145 & 47 & 2 & 168 & 109 & 56 & 3 & YES & 0.18 \\
\hline & Wright & & 1240 & 696 & 544 & & 1236 & 670 & 566 & & NA & NA \\
\hline & Zheng & & 229 & 137 & 77 & 15 & 184 & 103 & 63 & 18 & YES & 0.27 \\
\hline Q239H & & & $\mathrm{N}$ & GG & GT & TT & $\mathrm{N}$ & GG & GT & TT & & \\
\hline \multirow[t]{4}{*}{ (rs34677) } & Daugherty & 1 & 1136 & 854 & 265 & 17 & 1373 & 1038 & 316 & 19 & YES & 0.13 \\
\hline & & 2 & 101 & 84 & 15 & 2 & 394 & 341 & 47 & 6 & NO & 0.07 \\
\hline & Lee & & 194 & 143 & 46 & 5 & 168 & 123 & 43 & 2 & YES & 0.14 \\
\hline & Zheng & & 222 & 165 & 51 & 6 & 189 & 133 & 50 & 6 & YES & 0.16 \\
\hline
\end{tabular}

* number of data separately reported by articles; N: sample size; NA: not available; HWE: Hardy-Weinberg equilibrium; MAF: minor allele frequency 




1860 Asian Pacific Journal of Cancer Prevention, Vol 16, 2015 
There were 5 studies with 3292 cases and 3642 controls related to M9V polymorphism. M9V polymorphism plays a significant role in susceptibility of prostate cancer (Dominant Model: $\mathrm{OR}=0.87,95 \% \mathrm{CI}=0.78-0.97, \mathrm{P}=0.01$ ) (Figure 4). Effect of GG genotype on decreased prostate cancer risk was also indicated in subgroup with Caucasians (Dominant Model: OR=0.87, 95\%CI=0.77-0.97, $\mathrm{P}=0.01$ ), population-based controls (Dominant Model: $\mathrm{OR}=0.86$, 95\% CI $=0.77-0.97, \mathrm{P}=0.01$ ), quality control (Dominant Model: OR=0.87, 95\%CI $=0.77-0.97, \mathrm{P}=0.01)$ and larger sample size ( $>500)$ (Dominant Model: $\mathrm{OR}=0.87$, $95 \% \mathrm{CI}=0.77-0.97, \mathrm{P}=0.02)$.

\section{S201L polymorphism (rs2287939)}

Four studies with 2884 cases and 3364 controls were included in our meta-analysis of S201L polymorphism. Overall, significant decreased prostate cancer risk was detected in Dominant Model (OR $=0.90,95 \% \mathrm{CI}=0.81$ $0.99, \mathrm{P}=0.04)$. However, we identified no association between S201L polymorphism and prostate cancer risk when stratified by ethnicity, source of controls, quality control or sample size.

\section{K277E polymorphism (rs2278008)}

There were four studies with 1765 cases and 1984 controls involving in K277E polymorphism. The results indicated that K277E might not be related to prostate cancer risk in any five genetic model (Dominant Model: $\mathrm{OR}=0.89,95 \% \mathrm{CI}=0.78-1.02, \mathrm{P}=0.1)$. However, in subgroup analysis, $\mathrm{G}$ carrier have a decreased prostate risk in studies without quality control (Additive Model: $\mathrm{OR}=0.76,95 \% \mathrm{CI}=0.60-0.98, \mathrm{P}=0.3$ ). We didn't detect any association between K277E polymorphism and

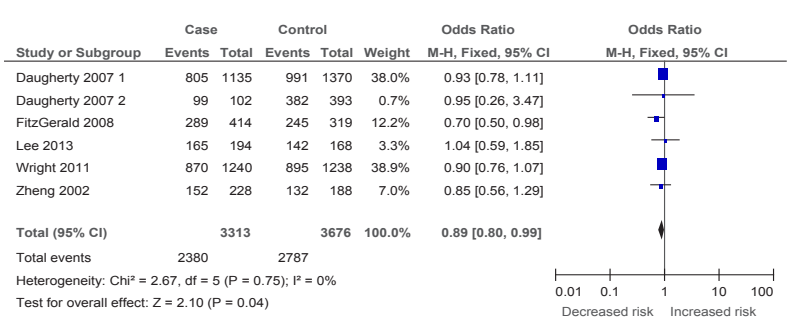

Figure 2. Forest Plot for the Association between the AMACR D175G Polymorphism and Prostate Cancer Risk (AG+GG vs AA). Significant association was observed between the $\mathrm{D} 175 \mathrm{G}$ polymorphism and prostate cancer risk

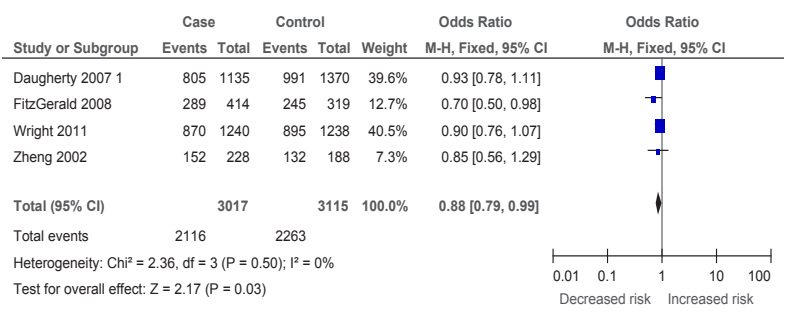

Figure 3. Forest Plot of Subgroup Analysis for the Association between the AMACR D175G polymorphism and Prostate Cancer Risk among Caucasians(AG+GG vs AA). We observed significant association between the $\mathrm{D} 175 \mathrm{G}$ polymorphism and prostate cancer risk among Caucasians

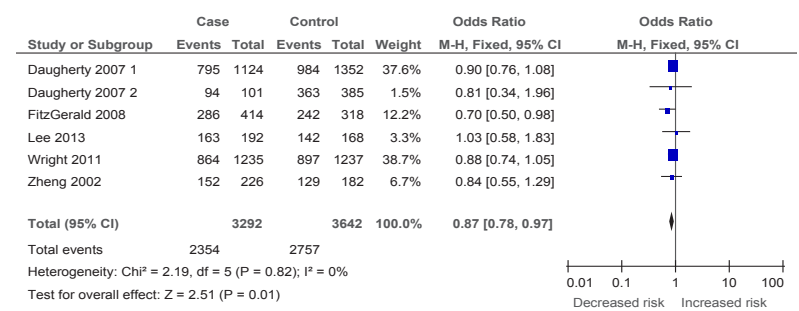

Figure 4. Forest Plot for the Association between the AMACR M9V Polymorphism and Prostate Cancer Risk (AG+GG vs AA). Significant association was observed between the M9V polymorphism and prostate cancer risk

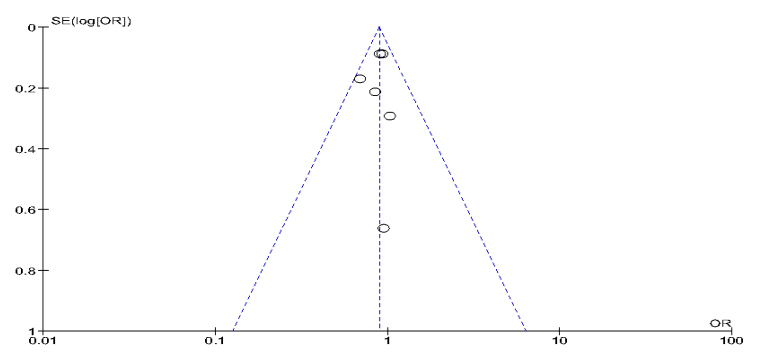

Figure 5. Begg's Funnel Plot on Publication bias for Included Studies on the Association of the AMACR D175G Polymorphism with Prostate cancer risk (AG+GG vs AA). The funnel plot seemed symmetrical, indicating absence of publication bias

susceptibility of prostate cancer in any other subgroup analysis.

\section{Q239H polymorphism (rs34677)}

Three studies recruiting 1552 cases and 1730 controls investigated the association between Q239H polymorphism and prostate cancer risk. No association was observed in any of the five genetic model (Dominant Model: $\mathrm{OR}=0.99,95 \% \mathrm{CI}=0.84-1.16, \mathrm{P}=0.88)$. In further subgroup analysis, there might be no effect of Q239H polymorphism on susceptibility of prostate cancer in any subgroup.

\section{Publication bias and sensitivity analyses}

Moreover, of all comparisons among studied loci, we didn't detect any obvious asymmetry in Begg's funnel plot (Figure 5). No significant publication bias was found in Egger's test. In addition, by excluding every single study sequentially, we observed no significant variation of ORs in sensitivity analyses.

\section{Discussion}

It was widely believed that the carcinogenesis of prostate cancer was influenced by environmental exposure and genetic factors both. As an essential genetic factor, SNPs as well as their association on cancer risk were researched extensively these years. Alpha-methylacylCoA racemase (AMACR) played a role in metabolism of branched-chain fatty acids and its abnormal expression was identified in prostate cancer. AMACR gene polymorphisms might affect the expression of enzyme and contributed to prostate cancer risk ultimately. A growing number of studies (Zheng et al., 2002; Daugherty 


\section{Nan Chen et al}

et al., 2007; FitzGerald et al., 2008; Wright et al., 2011; Lee et al., 2013) in regard to the association between AMACR gene polymorphisms and prostate cancer were conducted whereas contradictory results were obtained. Here we presented the first meta-analysis investigating the association between the five common AMACR gene polymorphisms and prostate cancer. Although only five studies were included in our work, we didn't detect any between-study heterogeneity and fix-effects model was applied to all analyses. So that our results in this metaanalysis were still much valuable and especially figure out the direction of future research.

The meta-analysis provided evidence for relationship between five common polymorphisms (D175G rs 10941112, M9V rs3195676, S201L rs2287939, K277E rs2278008 and Q239H rs34677) and prostate cancer risk. Our study suggested that D175G and M9V polymorphisms might be risk factors for prostate cancer. In subgroup analysis, we didn't contain Asian or African subgroup and hospital-based subgroup for lack of complete information. For D175G and M9V polymorphisms, we detected significant association between these two polymorphisms and prostate cancer in most of the subgroups, especially in subgroups including studies with population-based controls, quality control or larger sample size $(>500)$. Those results strongly proved important role of D175G and M9V polymorphisms in prostate cancer. It might be important to focus on these polymorphisms for their potential application in diagnosis, prognosis and therapy strategy of prostate cancer. Actually, it seemed that these two SNPs show an extremely high LD value and it supposed to do the linkage disequilibrium analysis. We couldn't do that for lack of corresponding method of meta-analysis to linkage disequilibrium analysis yet. For S201L polymorphism, although no relationship with prostate cancer was found in any subgroup analysis stratified by ethnicity, source of controls, quality control or sample size, potential decreased prostate cancer risk was detected in Dominant Model. Thus results of S201L polymorphism should be applied with much caution and more well-designed studies were warranted. It is worth pointing out that in Lee's study (Lee et al., 2013) based on Asians, for both D175G and M9V polymorphisms, AG genotype might increase prostate cancer risk in both dominant model and heterozygote comparison, although no significant difference wes detected. These results indicated that there might be some different effect on prostate cancer risk between Caucasians and Asians. But our meta-analysis couldn't conducted subgroup analysis on Asians for only one study was included. More studies based on Asians were hoped.

For K277E and Q239H polymorphisms, we didn't observed significant association between them and prostate cancer. Furthermore, no relationship with these two polymorphisms and prostate cancer was detected either. Those results might be attributed to small number of studies involving in K277E and Q239H polymorphisms. Also, development of prostate cancer was a complicated process which was influenced by interaction with genetic factors and environment. A single polymorphism might only provide limited effect and couldn't be detected among small sample sizes. Thus, more well-designed studies with population-based controls, well quality control and larger sample size were warranted, especially among other races.

Limitations of our meta-analysis should be presented. First, only five studies were included in our meta-analysis. Thus we suggested more studies investigating AMACR polymorphisms and prostate cancer risk should be launched. Besides, subgroup analysis by other ethnicities like Asian or Africa is lacked for incomplete information. More studies among these ethnicities were warranted in the future. Finally, all publications were obtained from selected databases and some publications might be left out.

In summary, our meta-analysis suggested that D175G and M9V polymorphisms of AMACR gene might were related to prostate cancer. The S201L polymorphism might be potentially associated with prostate cancer risk to some extent. However, no association was observed between K277E or Q239H polymorphisms and prostate cancer risk.

\section{Acknowledgements}

We sincerely expressed our thanks to all the people who helped in this study.

\section{References}

Amankwah EK, Sellers TA, Park JY, (2012). Gene variants in the angiogenesis pathway and prostate cancer. Carcinogenesis, 33, 1259-69.

Askari F, Parizi MK, Jessri M, Rashidkhani B (2014). Dietary patterns in relation to prostate cancer in Iranian Men: A Case-Control Study. Asian Pac J Cancer Prev, 15, 2159-63.

Daugherty SE, Shugart YY, Platz EA, et al (2007). Polymorphic variants in alpha-methylacyl-CoA racemase and prostate cancer. Prostate, 67, 1487-97.

Doolan G, Benke G, Giles G (2014). An update on occupation and prostate cancer. Asian Pac J Cancer Prev, 15, 501-16.

Ferdinandusse S, Denis S, L IJ, et al (2000). Subcellular localization and physiological role of alpha-methylacyl-CoA racemase. J Lipid Res, 41, 1890-96.

FitzGerald LM, Thomson R, Polanowski A, et al (2008). Sequence variants of alpha-methylacyl-CoA racemase are associated with prostate cancer risk: a replication study in an ethnically homogeneous population. Prostate, 68, 1373-79.

Jemal A, Bray F, Center MM, et al (2011). Global cancer statistics. CA Cancer J Clin, 61, 69-90.

Jiang N, Zhu S, Chen J, Niu Y, Zhou L (2013). A-methylacylCoA racemase (AMACR) and prostate-cancer risk: a metaanalysis of 4,385 participants. PLoS One, $\mathbf{8}, 74386$.

Lee SJ, Joung JY, Yoon H, et al (2013). Genetic variations of alpha -methylacyl-CoA racemase are associated with sporadic prostate cancer risk in ethnically homogenous Koreans. Biomed Res Int, 2013, 394285.

Lloyd MD, Yevglevskis M, Lee GL, et al (2013). alphaMethylacyl-CoA racemase (AMACR): metabolic enzyme, drug metabolizer and cancer marker P504S. Prog Lipid Res, 52, 220-30.

Mahmoud AM, Yang W, Bosland MC (2014). Soy isoflavones and prostate cancer: a review of molecular mechanisms. $J$ Steroid Biochem Mol Biol, 140, 116-32.

Mazzola CR, Ghoneim T, Shariat SF, (2011). Emerging biomarkers for the diagnosis, staging and prognosis of prostate cancer. Prog Urol, 21, 1-10.

Ouyang B, Leung YK, Wang V, et al (2011). Alpha-Methylacyl- 
CoA racemase spliced variants and their expression in normal and malignant prostate tissues. Urology, 77, 249. e1-249.e7.

Wang YC, Xia SJ (2009). An update of biomarkers in prostate cancer tissue. Zhonghua Nan Ke Xue, 15, 1039-43.

Wright JL, Neuhouser ML, Lin DW, et al (2011). AMACR polymorphisms, dietary intake of red meat and dairy and prostate cancer risk. Prostate, 71, 498-506.

Yang B, Chen WH, Wen XF, Liu H, Liu F (2013). Role of DNA repair-related gene polymorphisms in susceptibility to risk of prostate cancer. Asian Pac J Cancer Prev, 14, 5839-42.

Zhang LL, Sun L, Zhu XQ, et al (2014). rs10505474 and rs7837328 at 8q24 cumulatively confer risk of prostate cancer in Northern Han Chinese. Asian Pac J Cancer Prev, 15, 3129-32.

Zha S, Ferdinandusse S, Denis S, et al (2003). Alpha-methylacylCoA racemase as an androgen-independent growth modifier in prostate cancer. Cancer Res, 63, 7365-76.

Zheng SL, Chang BL, Faith DA, et al (2002). Sequence variants of alpha-methylacyl-CoA racemase are associated with prostate cancer risk. Cancer Res, 62, 6485-8. 